\title{
Aristóteles y el principio de individuación
}

\author{
Aristotle and the principle of individuation \\ Cindy Paola Lancheros Conde \\ Universidad Nacional de Colombia, Argentina \\ clancherosc@unal.edu.co
}

(D) https://orcid.org/0000-0002-1165-6262

\begin{abstract}
Resumen:
El propósito de este escrito es, a partir del análisis de Metafísica Z 1034a5-9, establecer cuál es el principio de individuación para Aristóteles. A fin de cumplir este cometido, el texto se encuentra dividido en cuatro secciones. En la primera de ellas presentamos, grosso modo, en qué consiste el principio mencionado y los candidatos al mismo. En la segunda, exponemos a la materia (hyle/ü $\lambda \eta$ )

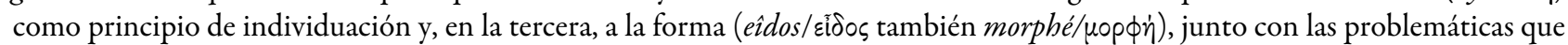
conlleva cada perspectiva. Para finalizar, proponemos un modo alternativo de entender a la forma como principio de individuación y a la materia como condición necesaria para el mismo.
\end{abstract}

Palabras clave: Individuo, Principio de individuación, Forma, Materia, Aristóteles.

\section{Abstract:}

The purpose of this paper is, from the analysis of Metaphysics Z 1034a5-9, to establish what is the principle of individuation for Aristotle. In order to fulfill this task, the text is divided into four sections. In the first of them we present, grosso modo, what the mentioned principle consists of and the candidates for it. In the second, we expose matter (byle/vi $\lambda \eta$ as a principle of individuation

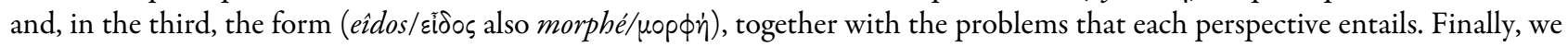
propose an alternative way of understanding form as a principle of individuation and matter as a necessary condition for it.

KEYWORDS: Individual, Principle of individuation, Form, Matter, Aristotle.

\section{SOBRE EL PRINCIPIO DE INDIVIDUACIÓN EN ARISTÓTELES}

En Metafísica Z8 encontramos una consideración sobre lo que en filosofía moderna se conoce como el principio de individuación. En palabras de Lloyd, un individuo se entiende como "lo que puede ser referido por un pronombre singular e identificado por un adjetivo demostrativo" (1970, p.519) ${ }^{1}$. Para Whiting, en cambio, "ser un individuo es simplemente ser una cierta forma" (1986, p.373). En términos generales, un individuo es una unidad indivisa e indivisible, que posee atributos o características que, en su totalidad, solo pueden ser atribuidas a él mismo; por ello es algo irrepetible, uno en número, y posee una única ubicación en el espacio-tiempo. En este sentido, un individuo es distinto a un particular, en cuanto este último es solo una instanciación de un universal con una multitud de réplicas idénticas. ${ }^{2}$ Ahora bien, aunque Aristóteles no plantea de manera explícita qué entiende por individuo, sí demuestra una preocupación por aquello que es uno en número y por la multiplicidad de sustancias: "[...] puesto que el esto y la cantidad no son lo mismo, no se llega a explicar cómo y por qué son muchas las cosas que son [...]" (Met. N2 1089b 30-32; trad. Calvo, 1982; énfasis agregado). Al preguntar sobre la distinción (cómo y por qué) de estas muchas cosas, se haya emprendido o no en su búsqueda, Aristóteles está señalando la necesidad de un principio de individuación, pues solo así puede dar cuenta de la multiplicidad numérica y su fundamento. El principio en cuestión, como consecuencia, busca dar respuesta a qué es lo que hace que una cosa sea distinta individualmente (numéricamente) de otra. 
Algunos estudiosos han estimado que Aristóteles sí da respuesta a lo que considera principio de individuación. Para tal afirmación se valen de cómo Aristóteles -de manera hipotética- podría haber respondido, si bien en sus escritos no hay una perspectiva clara o una postulación manifiesta del principio. Uno de los pasajes en los que se apoyan, y en el que nos centraremos en este escrito, es el siguiente:

[...] Basta con que el generante produzca y sea la causa de la forma en la materia. Solo entonces el todo esta forma precisa en estas carnes y huesos precisos, Calias y Sócrates, distintos gracias a la materia (pues es distinta) pero idénticos específicamente (pues la especie ${ }^{3}$ es indivisible) (Met. Z8 1034a5-9; trad. Correa, 2017). ${ }^{4}$

A partir de esta cita puede desprenderse una concepción según la cual la materia actúa como el principio de individuación. Sin embargo, algunos han considerado que este no es caso, sino que, es a la forma a quien debe atribuírsele este papel. Veamos más a fondo ambas posturas.

\section{MATERIA COMO PRINCIPIO DE INDIVIDUACIÓN}

La posición de que la materia es el principio de individuación aristotélico ha sido la más común en la tradición. Esto desde el medioevo, pasando por un simposio de la Sociedad Aristotélica a mediados del siglo XX, hasta la actualidad. Uno de los más grandes representantes de esta postura es Anthony Lloyd, quien, además de defender a la materia como principio de individuación, niega que la forma pueda serlo. El autor indica de manera explícita: "Aristóteles creía que el principio de individuación era la materia" (ibid. p.519).

Desde el punto de vista tradicional lo que significa 1034a5-9 es que, si bien Calias y Sócrates hacen parte de una misma especie, solo difieren en virtud de su materia. En efecto, reconocemos que Calias y Sócrates son trozos de materias distintos y, por ello, sabemos que no son una misma cosa, pese a que ambos son seres humanos. En este caso es la materia la que nos suministra información de que ambos sujetos son disímiles y uno en número, es decir, es ella la que actúa como principio de individuación. En contraste, la forma se limita a mostrar la igualdad de la especie, por lo que no es capaz de individuar.

Sin embargo, ni Charlton (1972) ni Cohen (1987) aceptan que el pasaje citado refiera a una diferencia numérica, sino tan solo a una cualitativa. Esto quiere decir que, para ellos, aquí no se está concibiendo a la materia como principio de individuación. Cuando Aristóteles afirma que "Calias y Sócrates, < [...] serán> distintos gracias a la materia” (Met. Z8 1034a8; trad. Correa, 2017), solo profiere que serán distintos cualitativamente, o, en otras palabras, que "Calias y Sócrates, que tienen la misma forma, tendrían diferentes características accidentales a causa de la materia que cada uno tiene” (Zárate, 2018, p.11). Dado que lo que da respuesta a la diferencia numérica no tiene que ser igual a lo que responde a la cualitativa, parece que no podemos concluir que la materia sea el principio de individuación, o por menos no podemos hacerlo a partir de la cita de Z8.

A pesar de lo planteado por Cohen, él reconoce que en Z8 sí se trata el tema de la diferencia numérica. Esto en 1033b29-32: "En algunos casos es también evidente que el generante es tal cual el generado, si bien no son una y la misma cosa numéricamente, sino solo en cuanto a la forma" ${ }^{5}$ (trad. Calvo, 1982; énfasis agregado). A partir de esto puede sugerirse que el asunto no es ajeno al capítulo y, que, por tanto, en 1034a5-9 podría existir alguna relación con el principio de individuación.

Así pues, admitiendo que aquí sea válido darle a la materia la capacidad de individuar, pueden dilucidarse las diferencias entre Sócrates y Calias en lo que a esto respecta. Por ejemplo, podemos afirmar que Sócrates tiene una estatura más baja que Calias, que tiene nariz chata, o que tiene ojos cafés oscuros; mientras que, Calias es narigudo y tiene ojos color miel. En este sentido, Sócrates y Calias parecen ser dos hombres numéricamente distintos. Sin embargo, la cuestión es más difícil al enfrentarnos a casos en los que no es posible distinguirlos de este modo, por ejemplo, cuando X y Y son iguales no solo en especie, sino también en su materia. Podemos plantear de manera hipotética que X y Y son gemelos y que, por tanto, su materia es indistinguible en lo que respecta a su cualidad. ${ }^{6}$ Si bien vemos que se trata de dos trozos materiales 
discontinuos y, por eso, uno es $\mathrm{X}$ y el otro $\mathrm{Y}$, la materia no nos dice nada acerca de qué hace a $\mathrm{X}$ y a $\mathrm{Y}$ ser lo que son. Por consiguiente, ella no parece dar respuesta a qué es lo que hace que dos individuos sean distintos, a menos de que se trate de una solución débil y superficial que apele a la percepción de dos materias singulares y discontinuas. Vinculando esto con un tema anterior, podemos afirmar que la materia da más cuenta de la disparidad entre particulares que entre individuos. ¿Pero es esto suficiente para caracterizarla como el principio de individuación aristotélico?

Es posible señalar un problema adicional en esta interpretación. Este radica en que, al ser la materia algo indeterminado en su totalidad, no es posible que ella determine ni individúe alguna cosa. La materia es mera potencialidad, con la capacidad de ser o no ser, pero incapaz de actuarse por sí sola; no puede caracterizarse ni siquiera como un esto. Nos dice Aristóteles:

Llamo materia a la que por sí misma no es algo determinado, ni un cuánto, ni ninguna otra con las que se determina lo que es. En efecto, hay algo de lo que cada una de estas se predica y cuyo ser es diferente al de cada una de las predicaciones (pues el resto se predican de la sustancia y ésta, de la materia) (Met.Z3 1029a20-24; trad. Correa, 2017).

De ahí que resulte inadecuado concebir a la materia como el principio de individuación, pues no parece ser el candidato más idóneo para tal puesto. Ella se limita a brindarnos, cuando está en el compuesto, una distinción superficial entre seres que poseen materias distintas, pero no nos dice nada acerca de la individualidad de los mismos. Siguiendo a Regis, "la materia, en sí misma sin características, no puede ser la causa de diferencias entre otras cosas. Si algo parece ser la materia es precisamente lo que requiere individuación" (1976, p.162).

Aun así, dentro de Metafísica $Z$, esta no es la única caracterización de la materia que encontramos. De modo curioso, en Z9 se aborda una nueva concepción según la cual "[...] < la materia es>, en un caso, capaz de moverse por sí misma [...] de manera determinada” (1034a13-14; trad. Correa, 2017). A esto se le conoce como materia próxima, la cual posee, en sí misma, alguna determinación, es decir, contiene parte de la forma que va a ser transmitida a lo generado. En este contexto se nos habla de la generación espontánea, ${ }^{7}$ la cual puede darse en virtud de este tipo de materia. Un ejemplo de este tipo de generación, usualmente considerado aristotélico, es cuando a partir de la carne descompuesta surgen gusanos. Aristóteles nos diría que la carne en cuestión posee la forma de gusano, y es por eso que estos animales se generan. A diferencia de la generación natural, en la cual solo a partir de gusanos se engendran gusanos, en la generación espontánea solo es necesaria la materia próxima para producir tales animales invertebrados. De este modo, la materia no se muestra como algo siempre indeterminado, pues incluye cosas que van más allá de sí misma. Desde este rasgo de la materia podría apelarse a que ella no necesita de la forma para ser lo que es, y de ahí que pueda postularse como principio de individuación. Sin embargo, la única característica resaltable de la materia próxima es que es capaz de generar con independencia de la forma, ya que la posee con anterioridad. ${ }^{8}$ Esto, aunque es bastante novedoso y desafía la concepción tradicional de la materia, no le da ventaja por encima de la forma. La materia próxima puede generar, pero no individuar.

Saliéndonos un poco de Aristóteles y trasladando la idea de la materia próxima a la actualidad, podemos esbozar una dificultad al respecto. Esta materia, en Metafísica Z, se exhibe como el sustento de la generación espontánea. No obstante, tal clase de generación ha sido refutada por varios estudiosos como Francesco Redi (1668), Lázaro Spallanzani (1769) y Louis Pasteur (1861). La carne no es poseedora de la forma de gusano ni puede producirla de la nada. Los gusanos surgen de huevos o larvas dejados por los insectos en la carne en descomposición. Desde luego, todo esto era desconocido para Aristóteles y de ahí que él pensara que la materia estaba calificada para semejantes logros. En todo caso, una vez evidenciado esto, parece que la materia no tiene supremacía alguna y que, poseyendo o no parte de la forma de una cosa, no es un elemento que sea definitivo o suficiente para la individuación.

Evidenciado esto, pasemos ahora a los que opinan que la forma debería ser el principio de individuación aristotélico. 


\section{FORMA COMO PRINCIPIO DE INDIVIDUACIÓN}

A partir del pasaje en cuestión, los que sostienen que la forma es el principio de individuación no tienen mucho más que decir, salvo negar que la materia pueda cumplir este rol, o bien, apelar a que la cita no le confiere alguna preponderancia a la materia. Esto, no obstante, no nos lleva a afirmar que la forma sí sea tal principio; de ahí que deban apelar a otros textos o fragmentos aristotélicos para respaldar su opinión. El mayor representante de esta postura es William Charlton, de quien, en la sección anterior, expusimos algunos argumentos en contra de la materia.

En primer lugar, los partidarios de la forma niegan que ella pueda entenderse solo como universal o especie. Pues, para poder actuar como un principio de individuación lo que se necesita ser es algo singular y propio de cada individuo, y el mero universal no basta para explicar todas estas peculiaridades. Por ello, los que sostienen esta postura argumentan que la forma es algo particular y determinado, esto es, un tóde ti ( $\tau$ ó $\varepsilon \tau \imath$ ). Así, aunque todos los hombres hagan parte de una misma especie y su forma o esencia sea el alma (cfr. Met. Z101035b13-14), ésta no tiene que ser igual en todos los individuos. Sócrates y Calias, en cuanto humanos, tienen un alma que es numéricamente distinta en cada caso y "[...] <el alma> de cada uno es cada uno <de ellos>” (Met.Z101035b18; trad. Correa, 2017). En este sentido, si bien todos los animales tienen alma y ésta es su esencia y su principio ( $c f r$. $D A$ I.1 402a6), cada uno tiene su propia alma particular que lo individua y lo hace distinto a los demás.

Entender la forma como particular tiene importantes consecuencias a la hora de declarar que 1034a5-9 da respuesta al principio de individuación aristotélico. Así pues, aunque se diga que Calias y Sócrates son iguales en especie, esto no es excluyente con la idea de que la forma de Calias y la de Sócrates son algo distinto. En efecto, en 1071a26-29 Aristóteles reconoce que puede haber individuos que, si bien son iguales en especie, son distintos en forma:

Y para las cosas de la misma especie son distintos, pero no específicamente, sino que son distintos individualmente: tu materia, tu forma ${ }^{9}$ y lo que en tu caso produce el movimiento y los míos, si bien son los mismos universalmente hablando" (Met. $\Lambda$ 5; trad. Calvo, 1982; énfasis agregado).

En concordancia con ello, pese a que 1034a5-9, en sí mismo, no afirme que la forma es el principio de individuación, tampoco lo niega. En relación con ello, los defensores de la forma también pueden apelar a que, el tema del capítulo, que constituye un conjunto con Z7 y Z9, se enmarca en el principio de sinonimia. El propósito de Aristóteles en este punto es apoyar su tesis de que lo que genera tiene la misma forma, en cuanto especie, que lo generado. De este modo, un hombre nace de hombres, y no de vacas o de pingüinos. El hombre que nace, empero, no es idéntico al hombre que lo engendra, pues son seres distintos. Es por ello que Aristóteles dice que se distinguen por su materia, con lo que solo busca resaltar el proceso de generación, mas no acudir a la materia como -o por lo menos el único- principio de individuación.

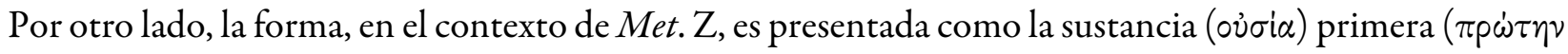

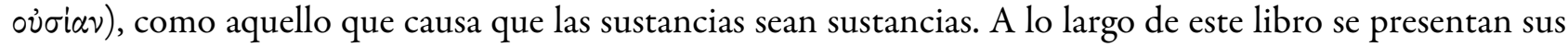
principales características, a saber, que es propia de cada cosa y no le pertenece a otra, que es un tóde ti, que está dotada de subsistencia o que es separada ( $\chi \omega \rho$ p $\tau \tau \dot{\delta} \nu)$, que es simple, y que no se dice de un sustrato. Además, no puede ser universal, pues "[...] parece imposible que cualquiera de las cosas dichas universalmente sea sustancia” (Met.Z13 1038b9; trad. Correa, 2017). Por este motivo, parece más razonable indicar que es la sustancia primera la que actúa como principio de individuación de las otras sustancias, que atribuir este papel a algún tercero o a las sustancias mismas.

Sin embargo, esta concepción de la forma está sujeta a algunas problemáticas. Para empezar, la equiparación entre tóde ti y particular no es del todo evidente. Según Gill (1994, p.68), la expresión tóde ti tiene dos acepciones. La primera de ellas se aplica a frases como "un cierto hombre", es decir, indica que hay "un cierto X". En la otra, tóde es demostrativo y $t i$ indica el sujeto; en nuestro ejemplo, "este hombre" o "este X". 
Ambas interpretaciones pueden entenderse como un tipo de cosa (hombre) o como una muestra ("token") particular, pero ninguna de ellas nos lleva a aceptar que la forma, en cuanto tóde ti, sea un particular o que sea capaz de individuar. Además, como señalan Frede y Patzig (1988, p.15), "no se puede excluir que Aristóteles permitiera que ambas concepciones coexistieran y las utilizara en contextos diferentes”, de modo que no es claro cuál debe atribuírsele a la forma.

Otra problemática se encuentra en 1071a26-29, antes citado. Pues allí no se afirma que la forma sea particular, sino que ella es algo distinto porque se encuentra encarnada en individuos disímiles. X y Y, pese a que hagan parte de una misma especie, supongamos humana, tienen materia y forma diferente con referencia a ellos mismos. Para comprender este punto es importante tener en cuenta que, antes de estas líneas, se estaba hablando de las causas próximas. Entonces, la idea no es admitir formas particulares, sino vislumbrar que entre generado y generador hay una diferencia en su forma, no en especie, pero sí entre causa y efecto. La forma de $\mathrm{X}$ y $\mathrm{Y}$ es distinta porque ambos surgieron de diferentes progenitores, en cuanto cada padre individual tenía una forma que actuó como causa próxima para generar a X y Y. Por consiguiente, la forma no es necesariamente y en sí misma particular, mas se manifiesta en diversos individuos.

Para finalizar, es importante resaltar que el debate acerca de si Aristóteles creía que la forma era particular o universal es algo que no ha tenido una respuesta inequívoca. Sin embargo, el hecho de que sea plausible que los universalistas tengan razón pone en aprietos a los que afirman que la forma es el principio de individuación, ya que les arrebata su base más sólida. Con todo, hasta este punto, parece que no hay razones o pruebas absolutas ni indiscutibles para sostener que la forma o la materia sean o deban ser el principio de individuación aristotélico.

\section{Posible Respuesta a la APORía}

Podemos preguntarnos, entonces, qué es lo que individua a los sujetos. A diferencia de Regis (1976), no creemos que en Aristóteles no sea posible establecer un principio de individuación ni concebimos que esto haya sido una problemática ajena a este pensador. Más bien, lo que hay que buscar es una manera de evitar o resolver los problemas señalados antes.

Como se mostró previamente, ni la materia ni la forma por sí mismas resultan ser suficientes para la individuación. Debemos, entonces, fijarnos en la unión de ambas para ver qué resulta de ello. Tenemos un compuesto cuando materia y forma están determinadas, esto es, cuando cierta forma se imprime en cierta materia. En esta unión, empero, materia y forma juegan un papel distinto y una puede considerarse más prioritaria que la otra. En este caso es la forma, pues ella es la sustancia primera, es quien saca a la materia de su indeterminación, y es la causa de la unidad del compuesto y de la materia (cfr. Met.Z17 1041b7-9). La forma, así pensada, es la que nos permite decir que un individuo es distinto de otro, porque en su configuración con la materia dan lugar a algo determinado y único. Desde luego, afirmar que cada uno tiene su materia y su forma no nos lleva a asumir que éstas son singulares por sí mismas, pues es en la conformación del individuo que la forma se transforma en algo propio del mismo (también la materia), en su alma. Esto nos libraría -o por lo menos en parte- de la dificultad de considerar a la forma como algo universal o particular. Ella, además, nos brinda más información que la materia acerca de en qué consiste ser este o aquel individuo, pues, al permitir a las sustancias ser tales, va más allá de simples diferencias cualitativas o de trozos de materia disímiles y discontinuos.

La forma, no obstante, no podría individuar con independencia de la materia, porque ésta es una condición necesaria para tal fin. En otras palabras, la "[...] materia juega un papel importante en la capacidad de la forma para individuar” (Whiting, ibid. p.371). Olvidarse de la materia es quizá el mayor error de muchos de los que aseveran que la forma es el principio de individuación. Cuerpo y alma tienen una dependencia mutua, ${ }^{10}$ "el alma no es separable del cuerpo" (DA II.2 413a5), y "el alma no existe [o es] sin un cuerpo" (DA II.2 414a20). En este orden de ideas, el alma actúa esencialmente como principio de explicación de la vida y del 
movimiento del cuerpo; de ahí que no pueda existir sin ellos y sin ser su causa. El alma no puede ser tal sin el cuerpo y es lo que es en virtud de sus propiedades causales. De modo análogo, el cuerpo es solo cuerpo animado, y decimos que un cuerpo sin vida (sin alma) es cuerpo solo por homonimia. ${ }^{11}$ Esta es la máxima expresión del hilemorfismo ${ }^{12}$ aristotélico, que no debe dejarse de lado al momento de declarar un principio de individuación.

Por otro lado, cuando nos referimos a individuos consideramos opciones de vida, gustos, elecciones, y demás, que van más allá de la materia misma, pero que muchas veces no podrían darse sin ella. La complexión física, por ejemplo, dispone a una persona en un limitado grupo de cosas que podría o no elegir, y es su alma quien determina qué quiere hacer. Este aspecto físico, por supuesto, está expuesto a cambios y modificaciones de acuerdo con aquello a lo que el alma sea afín. Una persona puede entrenar su cuerpo en X y Y cualidad y ser más fuerte y musculosa y dedicarse al boxeo; otra puede no contar con tanta suerte y, aunque desee dedicarse a esta labor, no poder conseguirlo ${ }^{13}$ en pro de su materia. Ahora bien, aunque se desempeñe de manera negativa en el boxeo, el alma de la persona puede seguir anhelando esta actividad, haciendo ella un hobby o alguna labor secundaria. El caso contrario sería la persona que, aunque fuera perfecta para ello (tuviera la materia ideal), no se interesara y más bien se dedicara a otras actividades. Aquí se hace evidente que la materia no basta para determinar a un individuo, mientras que, la forma, apoyada en aquella, logra explicar al individuo a cabalidad, actuando de manera más precisa como principio de individuación.

En la sección dedicada a la materia brindamos un ejemplo de acuerdo con el cual X y Y son gemelos y es imposible individuarlos solo en virtud de su materia. Aquí podemos hacer una pequeña anotación al respecto. El componente material nos ayuda a identificar que X y Y son dos cuerpos separados, pero no nos explica su individualidad, ya que ésta es la labor del alma. Aunque sean iguales en especie y en materia y no podamos distinguirlos de modo sencillo, su alma es diferente. A partir de ella X y Y se presentan como individuos dispares con características diversas y peculiares, que no son discernibles a simple vista. Al cruzarnos en la calle con X y Y solo podríamos apelar a sus materias discontinuas para afirmar que no son la misma persona. $\mathrm{Y}$ esto es valioso, claro, y al verlos pensamos más en individuos que en simples particulares. Pero para entender, en realidad, a causa de qué no son uno ni réplicas exactas, la materia no basta; es allí donde toma lugar la forma y esclarece estas cuestiones.

Siguiendo a Charlton, como uno de los primeros en desafiar la concepción ortodoxa del principio de individuación, no queda más que indicar lo siguiente: "Al decir esto deberíamos, por supuesto, ir más allá de los comentarios de Aristóteles. Pero si queremos desarrollar su pensamiento más allá del texto, esta y no la dirección tradicional es, sugiero, la que debemos seguir" (ibid. p.249).

\section{REFERENCIAS}

Aristóteles (2010). Acerca del alma (DA). Traducción de Marcelo Boeri. Buenos Aires: Colihue.

Aristóteles (1970) Metafísica (Met.). Ed. Trilingüe. Traducción de V. García Yebra. Madrid: Gredos.

Aristóteles (1982). Metafísica (Met.). Traducción de Tomás Calvo. Madrid: Gredos.

Aristóteles (2017). Metafísica (Met.). Traducción de Alfonso Correa. No publicado.

Black, M. (1952). The Identity of Indiscernibles. Mind, 61(242), new series, 153-164. Recuperado de http://www.j stor.org/stable/2252291

Charlton, W. (1972). Aristotle and the Principle of Individuation. Phronesis, 17(3), 239-249. Recuperado de http:/ /www.jstor.org/stable/4181890

Cohen, S. M. (1984). Aristotle and Individuation. Canadian Journal of Philosophy, Suppl. 10, 41-65.

Frede, M. \& Patzig, G. (1988). Aristoteles, Metaphysik Z. Text, Übersetzung und Kommentar. Zwei Band. München: Verlag C. H. Beck. 
Gill, M. L. (1994). Individuals and Individuation in Aristotle. En T. Scaltsas, D. Charles, y M. L. Gill, Unity, Identity, and Explanation in Aristotle's Metahpysics (pp. 55-72). Oxford: Oxford University Press.

Lloyd, A. (1970). Aristotle's Principle of Individuation. Mind, 79(316), new series, 519-529. Consultado el 7 de diciembre de 2020, de http://www.jstor.org.ezproxy.unal.edu.co/stable/2252437

Regis, E. (1976). Aristotle's 'Principle of Individuation'. Phronesis, 21(2), 157-166. Recuperado de https://www.jsto r.org/stable/4181987

Whiting, J. (1986). Form and Individuation in Aristotle. History of Philosophy Quarterly, 3(4), 359-377. Recuperado de http://www.jstor.org/stable/27743783

Zárate, F. (2018). Reconsideración del principio de individuación en la filosofía aristotélica (tesis de maestría). Universidad de los Andes, Bogotá, Colombia.

\section{Notas}

1. La traducción de los textos en inglés y alemán es nuestra.

2. La distinción entre individuo y particular juega un rol importante a la hora de determinar algún principio de individuación. En efecto, más que a diferenciar entre meras instanciaciones de un universal, a lo que se apunta es a caracterizar y dar cuenta de algo que es único e irrepetible.

3. El término aquí referido es "eîdos/عî̉os", que bien puede traducirse como "forma" o "especie". Aquí preferimos entenderlo como especie, en concordancia con la identidad específica de la que se habla en el pasaje. Esta traducción, además, permite entender mejor la postura de los que conciben la forma como principio de individuación.

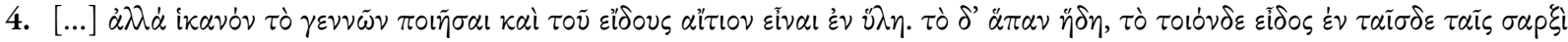

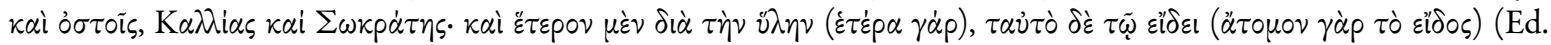
García, 1998).

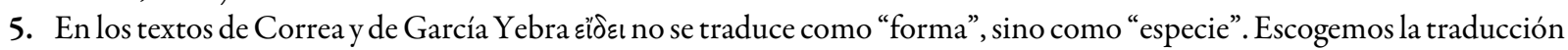
de Calvo porque es la más cercana a la de Ross, de quien Cohen extrae la cita.

6. El mismo caso se ilustra en el ejemplo de Max Black (1952, p.156), que consiste en suponer que en el universo existen solo dos objetos, dos esferas idénticas, hechas de hierro puro, con el mismo diámetro, color, temperatura, y demás. Siendo iguales, ¿qué permite distinguir a una de la otra?

7. Es importante resaltar que en otros textos Aristóteles es más explícito y detallado acerca de cómo se generan ciertos seres vivos a través de la generación espontánea, esto es, de cómo a partir de materia inerte se puede generar vida. Empero, estas consideraciones van más allá del objetivo del escrito. Para más información véase Generación de los animales.

8. Es importante tener en cuenta un matiz que presenta Aristóteles al respecto. Aunque en principio distintas, la forma y la materia próxima pueden verse como dos caras de una misma moneda. "[...] la materia última [próxima] y la forma son uno y lo mismo, aquello en potencia y esto en acto” (Met. H6 1045b18-19 trad. Calvo, 1982). Por lo que, bien sea en un caso u otro, el énfasis está puesto en la forma. La materia próxima es en potencia cierta forma de X; es su rol formal el que le permite ir más allá de sí misma.

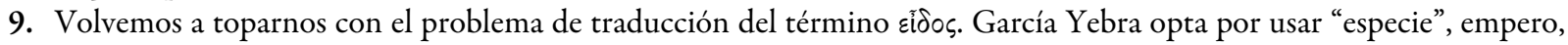
consideramos que entender a la especie como algo individual o disímil puede resultar problemático y confuso. En efecto, ¿cómo entender que, siendo ambos humanos, $\mathrm{X}$ tenga o haga parte de una especie individual y, por tanto, distinta a Y?

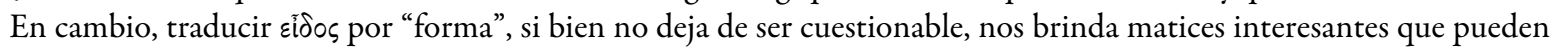
ser útiles para una discusión relativa al principio de individuación.

10. "Es forzoso, entonces, que el alma sea una sustancia en el sentido de forma de un cuerpo natural que en potencia tiene vida" (DA II.1 412a19-20).

11. "Pero el ojo es la materia de la vista; cuando la vista es removida, el ojo ya no es ojo, a no ser en sentido homónimo, como el [ojo] de piedra o el pintado. Hay que aplicar, claro está, lo dicho de la parte a la totalidad del cuerpo viviente" (DA II.1 412b20-23).

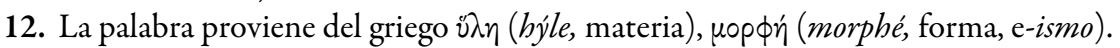

13. Aquí la idea de conseguir algo radica en poderlo hacerlo bien o de manera virtuosa. No sirve de nada dedicarse al boxeo si la persona fracasa en su intento o pierde todas las batallas. 